Gut, 1964, 5, 103

\title{
The constitution and properties of two gastrins extracted from hog antral mucosa
}

\section{Part I The isolation of two gastrins from hog antral mucosa}

\author{
R. A. GREGORY AND HILDA J. TRACY
}

From the Physiological Laboratory, the University of Liverpool

EDITORIAL SYNOPSIS This paper is the culmination of many years' work on the detection, isolation, physiological study, and now, large-scale production of gastrin. The final product has been identified as two almost identical peptides (gastrins I and II). Both are many times more potent than histamine in stimulating gastric acid secretion. Secretion may be inhibited if the dose administered is large. This duplex effect (stimulation in low concentration, inhibition in high concentration) has other pharmacological parallels such as the effect of nicotine on ganglionic transmission. This very important paper is likely to have many repercussions in clinical practice.

Attempts to isolate and characterize the antral hormone gastrin (Edkins, 1905, 1906) began with the work of Komarov $(1938,1942 a, b)$ and were continued by Uvnäs $(1943,1945)$, Munch-Petersen, Rönnow, and Uvnäs (1944), Harper (1946), and Jorpes, Jalling, and Mutt (1952). These studies established that there could be extracted from the antral mucosa of man, dog, cat, and hog a stimulant of gastric acid secretion which was probably of protein nature and was distinct from histamine. They provided some information concerning the chemical behaviour and physiological properties of the active prinicple; but the products obtained were relatively crude and the methods of extraction used were unsuitable for application on a large scale.

The method of extraction described by Gregory and Tracy (1961) made available for the first time a preparation which was shown to be highly active in stimulating gastric acid secretion when injected subcutaneously into conscious dogs. It was sufficiently pure to be given without ill-effects to a human subject. However, the procedure was tedious, expensive, and inefficient; and for these reasons it was unsuitable as a basis for isolation of the hormone in amounts sufficient for structural studies.

A new method of preparation has therefore been evolved; it can be worked easily and cheaply on a very large scale. The final product comprises two almost identical peptides, each in an apparently pure state. The physiological actions of these substances are discussed in Part II of this paper. A preliminary account of the work has been given to the Physiological Society (Gregory and Tracy, 1963).

\section{EXPERIMENTAL}

Hog antrums were brought from the abbattoir in batches of 300 . Delivery on the day of killing was not feasible; the antrums were everted and rinsed briefly in cold water at the time of collection and then refrigerated overnight. However, there was no significant loss of activity compared with smaller numbers of antrums obtained within two hours after killing. In the laboratory the antrums were boiled for 30 minutes in 60 litres of tap water and the mixture then cooled overnight. The liquor was drained off, clarified by adding coarse paper powder, and straining through fine nylon cloth, and the volume made up to 125 litres with cold tap water. In it was stirred for three hours $150 \mathrm{~g}$. of diethylaminoethylcellulose 'floc' (Whatman DE 50). The floc was then collected by filtration through fine nylon cloth and washed well with tap water. The gastrin, together with inert protein, was eluted by treating the floc with 2,000 to $2,500 \mathrm{ml} .0 \cdot 1$ $\mathrm{N} \mathrm{NaOH}$. Gastrin was found to withstand exposure to this solution for several hours at room temperature without significant destruction. The eluate was brought to $p \mathrm{H} 7$ with glacial acetic acid and then cooled to $10^{\circ} \mathrm{C}$. The $p \mathrm{H}$ was brought to 4 with glacial acetic acid, and, after refrigeration overnight, the precipitate was collected by suction filtration with the aid of acid-washed Hyflo Supercel. The cake of precipitate was stored at $-20^{\circ} \mathrm{C}$. until required. Activity was preserved without detectable loss for several weeks.

An amount of precipitate equivalent to 600 antrums was dissolved in water at $p \mathrm{H} 10$ by the addition of $18 \mathrm{~N}$ 


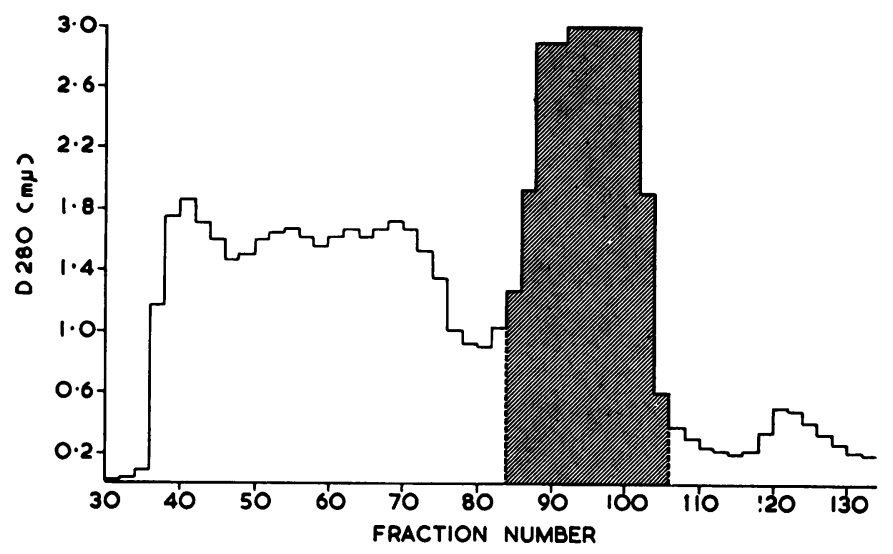

FIG. 1. Fractionation on Sephadex G50 of a gastrin extract equivalent to 600 antrums. The shaded peak (fractions 84 to 106) contained all the activity. ammonia solution, and the Hyflo removed by suction filtration. The volume of filtrate was made up to $600 \mathrm{ml}$. and in it (the temperature being maintained at 15 to $20^{\circ} \mathrm{C}$.) was dissolved $300 \mathrm{~g}$. of di-potassium hydrogen phosphate. Peroxide-free isopropanol (480 ml.) was then slowly added with vigorous stirring. After further stirring for $\mathbf{3 0}$ minutes, the mixture was centrifuged and the upper phase collected. An aqueous phase was recovered by adding 2 volumes of peroxide-free ethyl ether and $50 \mathrm{ml}$. water, shaking and centrifuging. The aqueous phase was extracted twice more with 2 volumes of ether and residual ether removed by vacuum aeration at room temperature.

The aqueous phase (at $10^{\circ} \mathrm{C}$.) was brought to $\mathrm{pH} 4$ with glacial acetic acid and after refrigeration overnight the precipitate was collected by centrifuging. It was dissolved in a volume of water equivalent to about half that of the original aqueous residue by adding a few drops of $18 \mathrm{~N}$ ammonia solution; precipitation at $10^{\circ} \mathrm{C}$. and $p \mathrm{H} 4$ was then repeated. After refrigeration for a few hours the precipitate was collected by centrifuging in a small cup (50 ml.) and dissolved in the minimal volume ( 3 to $5 \mathrm{ml}$.) by adding a drop or two of $18 \mathrm{~N}$ ammonia solution.

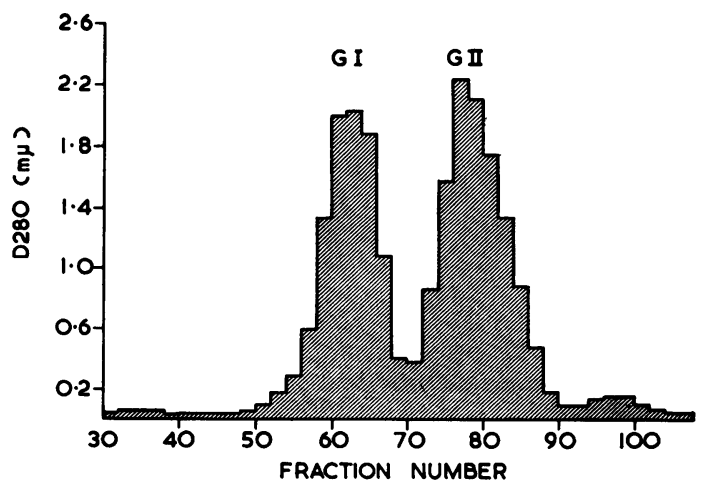

FIG. 2. Fractionation on an aminoethylcellulose column of the product obtained from the Sephadex fractionation (see Fig. 1). Fractions collected and pooled were 56 to 68 $(G I)$ and 72 to $88(G I I)$.
This solution was passed through a column (volume $700 \mathrm{ml}$.) of Sephadex gel (G 50 medium grade, Pharmacia) packed in $0.04 \mathrm{M}$ ammonium bicarbonate solution. Fractions of $5 \mathrm{ml}$. were collected and read at $280 \mathrm{~m} \mu$ in a spectrophotometer. Figure 1 shows a typical result; the major peak contained all the gastrin activity. The corresponding samples were pooled and dried in vacuo to constant weight; the final salt-free product weighed $54 \mathrm{mg}$.

This material was fractionated on a column $(1 \mathrm{~cm} . \times$ $30 \mathrm{~cm}$.) of aminoethylcellulose powder (Whatman AE 50) packed in 0.02 $\mathrm{M}$ ammonium bicarbonate solution. An approximately linear concentration gradient was arranged by supplying the column from a reservoir containing 0.2 $\mathrm{M}$ ammonium bicarbonate solution through a mixing vessel, volume $180 \mathrm{ml}$., containing the starting concentration $(0.02 \mathrm{M})$. Samples $(1.3 \mathrm{ml}$.) were collected every 15 minutes; they were diluted to $4 \mathrm{ml}$. with water and read at $280 \mathrm{~m} \mu$. Figure 2 shows the result; two major fractions (GI and GII) emerged almost completely separated, followed by a small inactive peak. The fractions corresponding to the central portions of each major peak were pooled and dried in vacuo. Both fractions were then re-run on similar AE columns, using gentler gradients $(0.05$ to $0.2 \mathrm{M}$ for $\mathrm{GI}$ and 0.06 to $0.3 \mathrm{M}$ for GII) and collecting larger samples $(2.5 \mathrm{ml}$. in 15 minutes); the volume of the mixing vessel was the same as before (180 ml.). The samples were read at $280 \mathrm{~m} \mu$; the results are shown in Figure 3. The appropriate samples were pooled and dried: the final products weighed $17 \mathrm{mg}$. (GI) and $22 \mathrm{mg}$. (GII).

At this stage both gastrins still contained a trace of pigment which could be removed by high-voltage electrophoresis, but were otherwise virtually pure. Amounts of 10 to $20 \mathrm{mg}$. were applied as a narrow band on thick paper (Macherey \& Nagel 214, or Whatman $3 \mathrm{MM}$ ) and run successively in (1) $0.1 \mathrm{~N}$ borate buffer at $p \mathrm{H} 9$ or pyridine-acetic acid-water $(10: 0.4: 90$ by volume) at $p \mathrm{H} 6 \cdot 3$, and (2) $15 \%$ acetic acid ( $p \mathrm{H} 2)$. After each run (approximately two hours) guide strips were cut from the sides and centre of the paper and stained with the Cl-imino reagent of Rydon and Smith (1952) as modified by Reindel and Hoppe (1954). The region of the main paper containing the gastrin was cut out, eluted with water or dilute ammonia solution, and the solution 


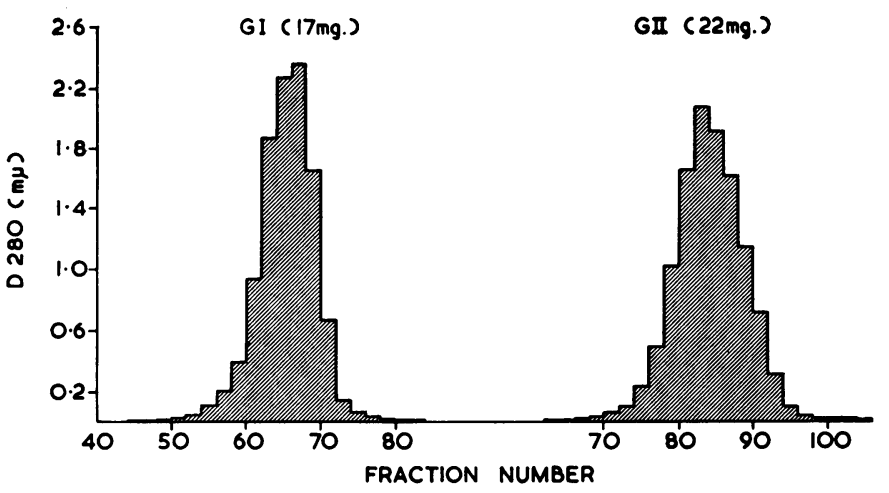

FIG. 3. Refractionation on aminoethylcellulose columns of GI and GII (see Fig. 2). Fractions collected and pooled were 58 to $72(G I)$ and 74 to 96 (GII). The final yields are shown. dried in vacuo. The voltage gradient in all runs was 40 to $50 \mathrm{v} . / \mathrm{cm}$. with appropriate refrigeration.

Successive electrophoresis in these systems did not alter either the amino-acid constitution or the physiological activities of either gastrin. The final products ran as single compact spots with nothing else detectable on the paper, using the Cl-imino reagent (Fig. 4). This is a stringent test for the presence of contaminants since, as Rydon and Smith showed, it reacts with all proteins, all peptides, most amino-acids, and many related compounds, and is very sensitive. Both gastrins were found to be ninhydrin-negative, indicating the absence of a terminal amino-group in the molecule. This conclusion was confirmed by a failure to demonstrate an N-terminal group using the fluorodinitrobenzene method (FraenkelConrat, Harris, and Levy, 1955).

Quantitative amino-acid analyses of both gastrins after the second fractionation on animoethylcellulose were made for us by Dr. R. C. Sheppard of the Department of Organic Chemistry of this University, using the Beckman-Spinco Autoanalyser, model 120B. Figure 5 shows a typical result for GII after acid hydrolysis. The peak labelled $X$, which we referred to in a preliminary communication (Gregory and Tracy, 1963) as 'an unidentified substance', has since been identified as tryptophane. The preservation of this amino-acid on acid hydrolysis is unusual, and is attributable to the complete absence of carbohydrate and metal ions from the preparation.

Both gastrins were found to have the same amino-acid constitution, viz., aspartic acid (1), glutamic acid (6), glycine (2), alanine (1), methionine (2), tyrosine (1), tryptophane (2), proline (1), phenylalanine (1). There was also present in each molecule one ammonia residue.

The minimal molecular weight for each gastrin, calculated from the amino-acid composition, is approximately 2114. Ultracentrifugal analysis (see Appendix) gave values for both GI and GII of approximately 1335 . The reason for this considerable discrepancy remains at present unexplained; but the results indicate that the true molecular weight of each gastrin is not a multiple of that calculated.

Both gastrins were many times more potent than histamine in stimulating gastric acid secretion when injected as single subcutaneous doses in a conscious dog provided with a completely denervated pouch of the gastric fundus (Fig. 6).

\section{DISCUSSION}

The object of the work reported here was to evolve a method for the preparation of gastrin which would provide a supply of the pure hormone ample for chemical and physiological studies. The final product has been shown to consist of two almost identical peptides each in an apparently pure state; they have an identical amino-acid constitution. Structural studies of both substances are in progress under the direction of Professor G. W. Kenner, in

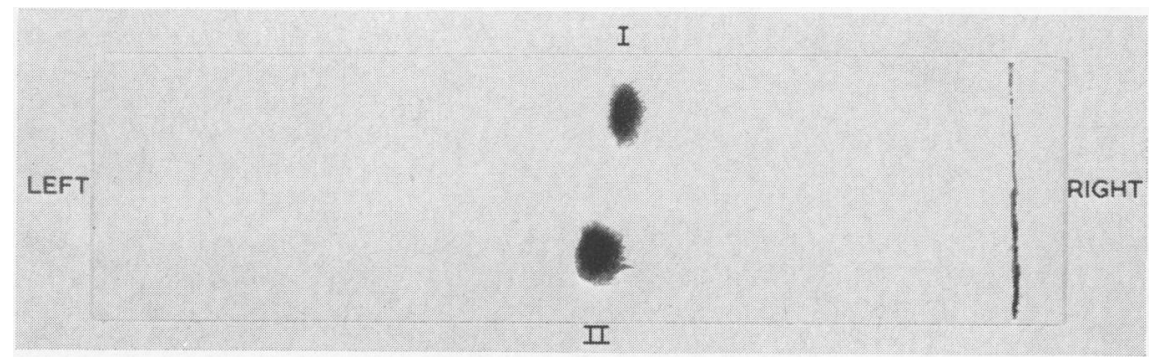

FIG. 4. Simultaneous paper electrophoresis of GI (above) and GII (below). Each spot contained about $50 \mu \mathrm{g}$. Origin (cathode) on right. Whatman No. 3MM paper. Borate buffer $(0 \cdot 1 N$ in $\mathrm{NaOH}$ ), pH 9.0. Gradient $20 \mathrm{v} / \mathrm{cm}$. Cl-imino stain. 


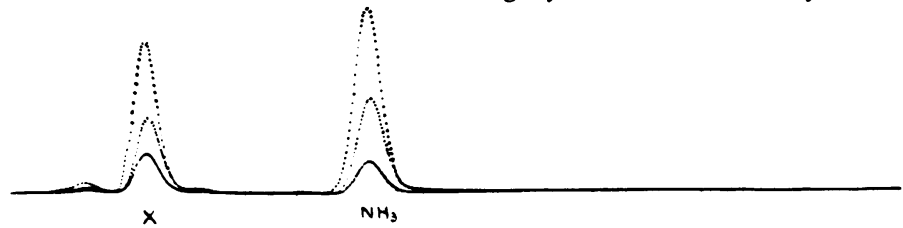

$x$
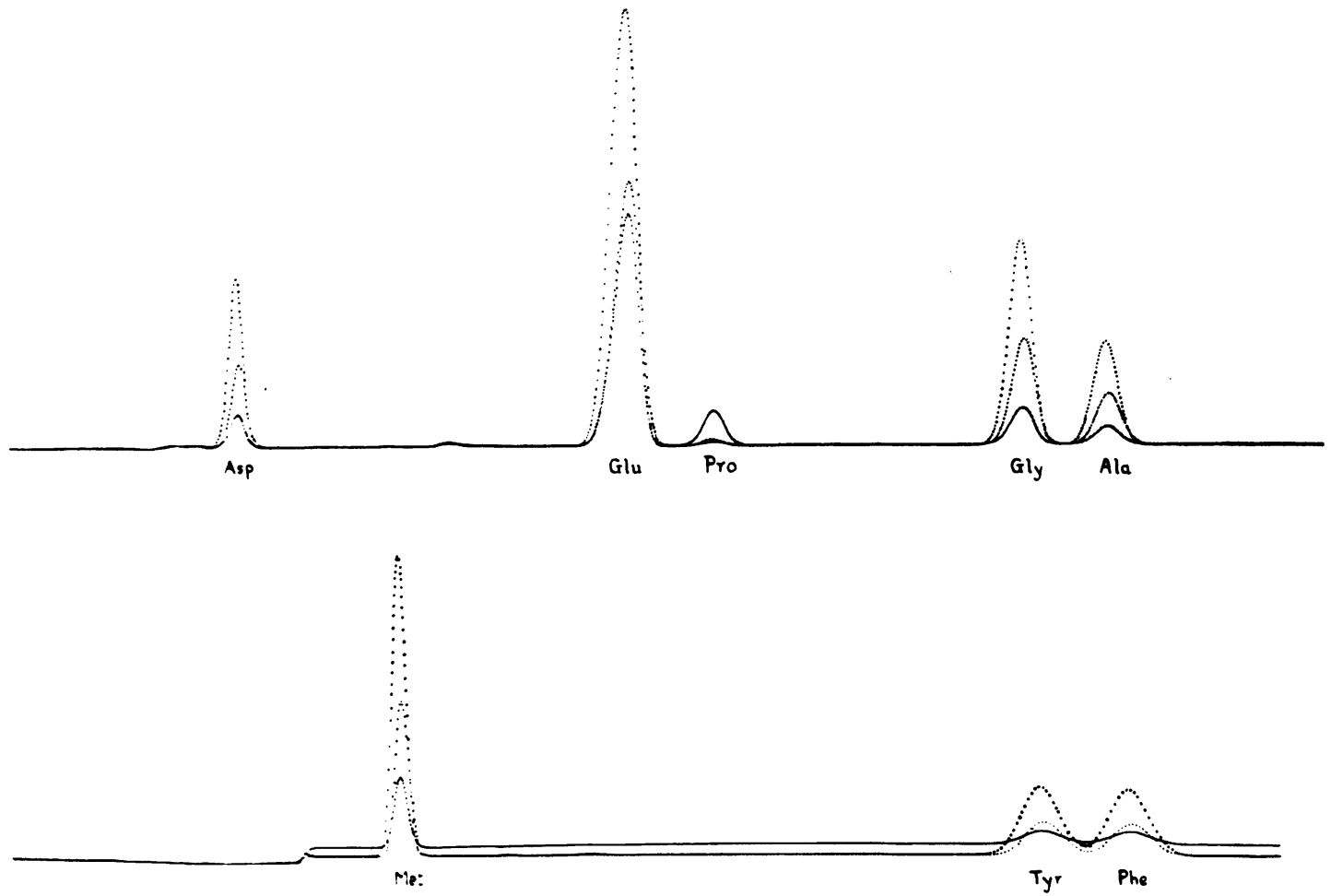

FIG. 5. Quantitative amino-acid analysis of an acid hydrolysate of GII after two fractionations on aminoethyleellulose. The peaks are: top, $X$ (tryptophane) and ammonia; middle, aspartic, glutamic, proline, glycine, alanine; bottom, methionine, tyrosine, and phenylalanine.

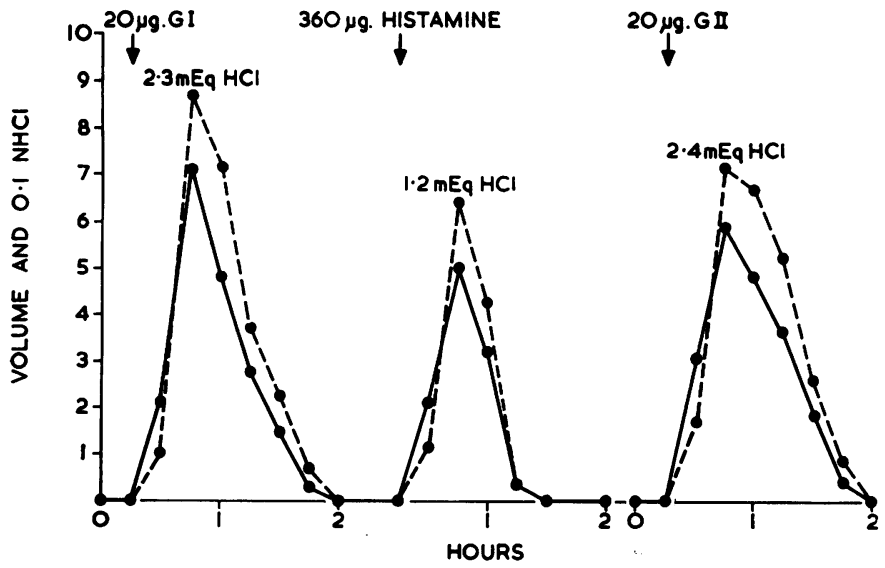

FIG. 6. Responses (on different occasions) of a conscious dog provided with a denervated pouch of the gastric fundus to the subcutaneous injection

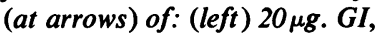
(centre) $360 \mu \mathrm{g}$. histamine base, and (right) $20 \mu \mathrm{g}$. GII. The total acid outputs $(m E q . \mathrm{HCl})$ for each response are shown. ..... - acid. __ volume. 
the Department of Organic Chemistry of this University, and the amino-acid sequence in the molecule of GII has been elucidated; the N- and Cterminal groups have been established as pyroglutamyl and phenyl-alanine-amide, respectively. Similar studies on GI are now well advanced; and although the end groups for this molecule have not yet been positively identified, present evidence indicates that they are the same as in GII. These conclusions are in agreement with the observations (see text) that no N-terminal group can be identified in either gastrin, using fluorodinitrobenzene, and that both are ninhydrin-negative. It thus appears highly probable that the different electrophoretic and chromatographic behaviour of the two molecules, which enables them to be separated, must depend on some structural feature within the body of the molecule.

The conclusion that both gastrins are pure is based upon the findings (1) that the quantitative aminoacid composition of each is consistent with that of a single molecule, (2) that the quantitative amino-acid composition of each remains unchanged after successive high-voltage electrophoresis in alkaline and acid buffer systems, (3) that ultracentrifugal analysis shows no evidence of inhomogeneity in either, and (4) that neither has an $\mathrm{N}$-terminal group demonstrable with fluorodinitrobenzene. Further support for the conclusion is to be found in the fact that during elucidation of the structure of GII there have come to light no inconsistencies which would suggest that the preparation was not homogeneous.

Both gastrins have been shown to be potent stimulants of gastric acid secretion when injected subcutaneously into conscious dogs provided with completely denervated fundic pouches; and preliminary studies made by Dr. W. I. Card and Dr. S. Mahklouf, of the Western General Hospital, Edinburgh, show that GII is many times more powerful than histamine in stimulating gastric acid secretion when injected subcutaneously into human subjects.

The substances described have been designated 'gastrin' I and II for convenience in description; but it is at present not known whether either is the form in which the antral hormone is released into the circulation, and whether both gastrins exist in the antral mucosa during life, or one is formed from the other during extraction or after death. Evidence on the latter point is being sought.

\section{Part II The properties of two gastrins isolated from hog antral mucosa}

The idea that there exists in the antral mucosa an hormonal stimulant of gastric secretion originated in the work of Edkins $(1905,1906)$ who injected crude aqueous extracts of gastric and intestinal mucosa intravenously into anaesthetized cats and reported that those made from the antral region (and also from the cardiac region of the hog stomach) contained a stimulant of gastric acid secretion which could not be found in significant amounts elsewhere.

Several groups of workers have since studied the physiological properties of antral extracts, usually in the course of attempts to isolate the principle responsible for the stimulation of gastric acid secretion. It has been shown that crude antral extracts, when injected intravenously into anaesthetized animals. exhibit some or all of the following actions: 1 stimulation of gastric acid secretion; 2 stimulation of pepsin secretion; 3 stimulation of pancreatic volume flow (slightly); 4 stimulation of pancreatic enzyme secretion; 5 stimulation of gastric and small intestinal tone and motility; 6 little or no stimulation of gall-bladder tone and hepatic bile flow; and, 7, inhibition of gastric acid secretion stimulated by some other means.

Edkins (1906) remarked that some of his gastrin extracts seemed to stimulate pepsin as well as acid secretion. Komarov (1942b) did not confirm this, but noted the presence in his crude extracts of a small amount of secretin activity, as also did MunchPetersen et al. (1944). The latter noted a slight stimulation of hepatic bile flow, but were unable to detect any effect on gastric motility. They did, however, observe, as had Uvnäs (1943), that the response to successive injections of the same extract frequently declined during the course of an experiment; and this observation was confirmed by Uvnäs (1945) and by Jalling and Jorpes (1947). The former suggested that it might be due to the presence of an inhibitor, possibly enterogastrone, in the preparation; the latter attributed it to exhaustion of the secretory mechanism, since it was most evident following a large dose of gastrin. Uvnäs (1948), extending his earlier observation (Uvnäs, 1945) that his gastrin extracts often showed a slight power to stimulate pepsin secretion, suggested that a pepsigogue principle, possibly of hormonal nature, was present.

Blair, Harper, and Lake (1953) reported in abstract form the presence in crude antral extracts of a stimulant of pepsin secretion which was apparently 